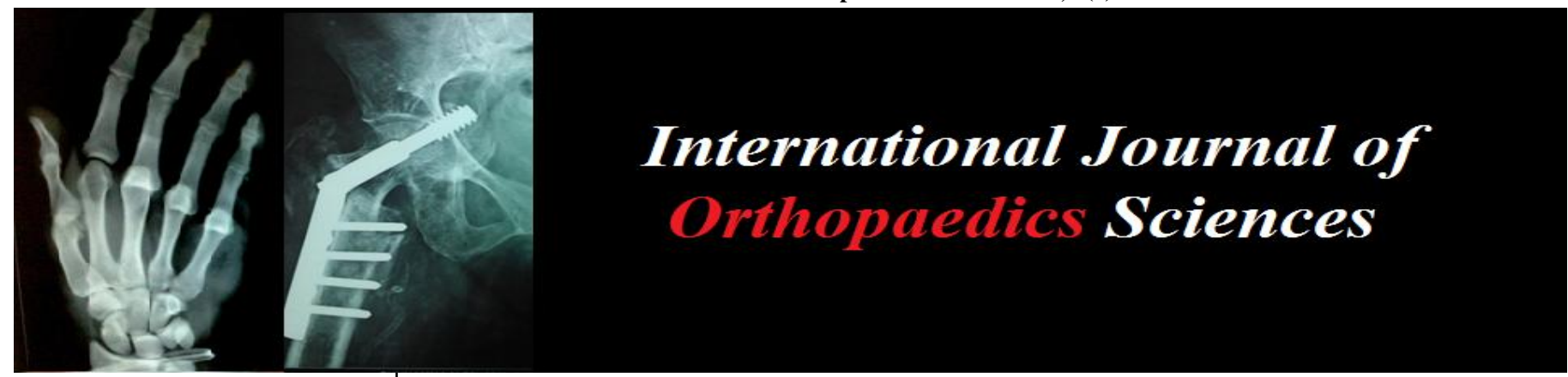

E-ISSN: 2395-1958

P-ISSN: 2706-6630

IJOS 2020; 6(2): 464-471

(C) 2020 IJOS

www.orthopaper.com

Received: 01-01-2020

Accepted: 03-02-2020

Dr. Deepak Shivanna

Professor and Unit Head,

Department of Orthopedics Bangalore Medical College and

Research Institute, Bangalore,

Karnataka, India

Dr. Prathamesh M Hanchnal

Assistant Professor, Department

of Orthopaedics, Jawaharlal

Nehru Medical College, Belagavi,

Karnataka, India

Dr. Abhishek Saxena

Senior Resident, Department of

Orthopaedics, Tejasvini

Hospital, Mangalore, Karnataka,

India
Corresponding Author:

Dr. Prathamesh M Hanchnal

Assistant Professor, Department

of Orthopaedics, Jawaharlal

Nehru Medical College, Belagavi,

Karnataka, India

\section{Clinical and radiological outcome of closed extra articular distal tibial (Pilon A) fractures treated by minimally invasive percutaneous plate osteosynthesis (MIPPO)}

\author{
Dr. Deepak Shivanna, Dr. Prathamesh M Hanchnal and Dr. Abhishek \\ Saxena
}

DOI: https://doi.org/10.22271/ortho.2020.v6.i2h.2082

\section{Abstract}

Background: Management of distal tibial fractures remains one of the therapeutic Challenges. Mechanically stable fixation can be obtained without significant dissection and surgical trauma to the bone and soft tissues by Minimally invasive percutaneous plate osteosynthesis (MIPPO) ${ }^{[1]}$

Objectives: 1) To study and evaluate the clinical and radiological outcome of pilon A (Extra articular) fracture with MIPPO technique using LCP and also study the complications

Methodology: Patients of Bangalore medical college fulfilling inclusion \& exclusion criteria be taken into study after informed written consent. Details including examination and investigations will be recorded in study Performa. MIPPO will be carried out depending on specific fracture pattern. Cases reviewed at 4,8,12 wks and 24 months both clinically and radiologically for bony union. Clinical outcome and Radiological criteria regarding fracture union and complications shall be documented. Functional outcome measured by LEFS score. Review of literature obtained and results compared with our study. Since these are proven methods of fixation, no control groups are required.

Results: Our study had 30 patients 19 male and 11 female. Average time for union was 16.4 weeks. Average time for full weight bearing was 18 weeks, 76 percent of our patients were back to previous work by 20 weeks. One patient had wound infection and his implant was removed and ex Fix applied, he needed plastic surgery intervention, and one patient had delayed union for Whom bone grafting was done by 10 weeks. 2 patients developed ankle stiffness.

Conclusion

1. Locking compression plate is a good system for Distal tibia (Pilon A) fractures.

2. As surgical dissection is minimum operating time is reduced.

LCP by MIPPO technique leads to less periosteal stripping and less softtissue damage, gives good angular stability and hence more rate of union and less chance of infection.

Keywords: Adiological, articular distal, minimally invasive percutaneous plate osteosynthesis

\section{Introduction}

Distal tibia often presents a challenge to orthopaedic surgeon. Destot first used the term In 1911 , likening the pilon to a pestle, the tibial pilon compromises anatomically the distal end of tibia, its proximal limit is found approximately $8-10 \mathrm{~cm}$ from the ankle articular surface, where triangular section of the tibial diaphysis, with its anterior crest, changes direction forming the metaphysis. The three dimensional configuration of this region appears to be designed to increase the area of the articular surface, reducing stress on the ankle joint ${ }^{[4]}$.

Tibial pilon fracture represents 5-7\% of all tibial fractures, these are usually the result of high energy axial compression and rotation forces. They are usually associated with severe soft tissue compromise. The limited soft tissue coverage, subcutaneous location, poor bone quality, osteoporosis renders the distal tibial fracture very challenging ${ }^{[5]}$. 
The difficulty that arises in the treatment of fracture distal tibia are -

1. The tendency to redisplacement of the fracture fragments following subsidence of swelling specially in oblique, spiral and comminuted fractures when treated by Cast.

2. Delayed union due to the precarious vascularity in distal tibia.

3. Functional and cosmetic deformation if rotational alignment or positon of the

4. fragment is not achieved, as it is important because knee and ankle joint are in same parallel axis.

External fixation can be a useful option in open fractures with soft tissue injury, but can lead to pin-track infections, septic arthritis and delayed union. Soft tissue management has been seen to play a vital role management alongside the bony reconstruction ${ }^{[8]}$.

Biological plate fixation technique are based upon the principles of limited soft tissue stripping, maintenance of osteogenic fracture hematoma and preservation of vascular supply to the individual fracture while restoring axial and rotational alignment and providing sufficient stability to allow progression of motion, uncomplicated fracture healing and eventual return to function. As such, the evolution of biological plating technique has lead to low profile, precontoured implants specifically intended for application in the distal tibia ${ }^{[10]}$.

\section{Aims and objectives}

1. To study and evaluate the clinical and radiological outcome of pilon A (Extra- Articular) fracture with MIPPO technique using LCP.

2. To evaluate the incidence of associated complications

\section{Materials and Methods}

This study is a prospective study done from Nov 2015 to May 2017 having 30 subjects, done in Bowring and lady courzon hospital and Victoria hospital both affiliated to BMCRI. Study comprised of 30 patients who had closed extraarticular distal tibial fractures who were operated with Distal tibial Locking plates using minimally invasive (MIPO) technique

\section{Inclusion Criteria}

- Age group of 16-65 years of either sex.

- Closed distal Tibial extra-articular fractures (Pilon A).

\section{Exclusion Criteria}

- Age <16 yrs and >65yrs. All open distal Tibial fractures, distal tibial intraarticular fractures. Segmental fractures. Patients with severe co morbidities. Pathological Fracture.

- Cases unfit for surgery due to co-morbid factors/ systemic diseases.

\section{Methodology}

Patients of Victoria Hospital and Bowring \& Lady Curzon hospital with closed extra articular distal tibial fractures who fulfill the inclusion \& exclusion criteria were taken in to study after obtaining informed written consent. Demographic data, History, Clinical examination and details of investigations were recorded in the study Performa. Minimally Invasive Percutaneous Plate Osteosynthesis was carried out depending on requirement for specific fracture pattern. These cases were reviewed periodically at $4,8,12$ weeks and 24 weeks both clinically and radiologically for bony union. Clinical outcome and Radiological criteria (RUST SCORE) regarding fracture union and complications was evaluated and documented. Functional outcome was measured by lower extremity functional score (LEFS).

Detailed review of literature available on this subject was studied and results obtained in my study were compared with results obtained in other simila studies. Since these are proven methods of fixation, no control groups are required.

\section{Surgical Technique}

Under regional or general anesthesia and patient in supine position involved leg was painted and draped. Tourniquet was applied and inflated before the start of procedure. If there is a associated fibula fracture, initial fibula fixation was done with $1 / 3^{\text {rd }}$ tubular plate, it restores the limb length and helps in reduction of tibial plafound ${ }^{50}$. A vertical or curvilinear incision was made with medial malleoli as reference and to take care not to injure the saphenous vein and nerve. Now a subcutaneous plane was made without stripping the periosteum and disturbing the fracture site. Fracture was reduced under $\mathrm{c}$ arm control and where reduction was difficult $\mathrm{k}$-wires were used as joystick to achieve the reduction and reduction forceps to hold the reduction. Pre countered DTLCP was passed into the plane created and its position confirmed under c arm. Plate was initially fixed with help of $\mathrm{K}$ wires, Before fixing the plate with screws sagging of distal fragment was prevented by using a towel behind it, position of plate was confirmed before placement of screws under $c$ arm in both AP and Lateral views. Provisional non locking screws used to bring plate near the bone. Interfragmentary screws used to achieve compression in simple fractures. With separate stab incisions atleast 3 locking screws were used on either side of fracture site under $c$ arm guidance ${ }^{11}$.Comminution - In comminuted fractures, the plate span ratio was kept to 3 and in case of fractures with a simple fracture configuration, a plate 8-10 times longer than the fracture was planned. Reduction was confirmed under $\mathrm{c}$ arm and wound was closed with absorbable subcutaneous sutures and skin closed with staples.

\section{Follow up}

Patient was followed up at 4 weeks, 8 weeks, 12 weeks and 24 weeks and protected weight bearing was allowed once callus formation was visible and gradually increased according to clinical and radiological signs of fracture healing. Fracture was considered united radiologically when visible callus was seen in atleast 3 cortices in antero-posterior and lateral views and clinically when there was no pain.

\section{Results}

Table 1: Distribution of Samples by Age

\begin{tabular}{|c|c|c|}
\hline Age (Years) & Frequency & Percentage \\
\hline $18-30$ & 3 & 10 \\
\hline $31-40$ & 5 & 16.66 \\
\hline $41-50$ & 9 & 30 \\
\hline $51-60$ & 6 & 20 \\
\hline $61-70$ & 7 & 23.33 \\
\hline Total & 30 & 100 \\
\hline
\end{tabular}

Table 2: Distribution of Sample by Sex

\begin{tabular}{|c|c|c|}
\hline Sex & Frequency & Percentage \\
\hline Male & 19 & 63.33 \\
\hline Female & 11 & 36.66 \\
\hline
\end{tabular}


Table 3: Distribution of Samples by Type of Fracture (AO Classification)

\begin{tabular}{|c|c|c|}
\hline Type(A043a_) & No of Cases & Percentage \\
\hline A1 & 6 & 20 \\
\hline A2 & 15 & 50 \\
\hline A3 & 9 & 30 \\
\hline
\end{tabular}

Table 4: Distribution of Samples According To Time Taken For Fracture Union

\begin{tabular}{|c|c|c|}
\hline Union (Weeks) & No of Cases & Percentage \\
\hline$<16$ & 11 & 36.66 \\
\hline 18 & 6 & 20 \\
\hline 20 & 6 & 20 \\
\hline 22 & 5 & 16.66 \\
\hline$>24$ & 2 & 6.66 \\
\hline
\end{tabular}

Table 5: Distribution of Samples According To Complications

\begin{tabular}{|c|c|c|}
\hline Complications & No of Cases & Percentage \\
\hline Wound infection & 1 & 3.33 \\
\hline Implant prominence & 1 & 3.33 \\
\hline Nonunion & 0 & 0 \\
\hline Malunion & 1 & 3.33 \\
\hline Delayed union & 1 & 3.33 \\
\hline Stiffness & 2 & 6.66 \\
\hline
\end{tabular}

Table 6: Distribution of Samples According To Lefs Score

\begin{tabular}{|c|c|c|}
\hline Lefs Score & No Of Cases & Percentage \\
\hline $20-40$ & 3 & 10 \\
\hline $40-60$ & 6 & 20 \\
\hline $60-80$ & 21 & 70 \\
\hline
\end{tabular}

Lefs- Lower Extremity Functional Score

Table 7: Distribution of Samples According To Rust Score

\begin{tabular}{|c|c|c|}
\hline Rust Score & No Of Cases & Percentage \\
\hline $4-6$ & 1 & 3.33 \\
\hline $6-9$ & 8 & 26.66 \\
\hline $9-12$ & 21 & 70 \\
\hline
\end{tabular}

Rust- Radiographic Union Scale In Tibia

\subsubsection{Results}

All the fractures operated in our study were extra-articular fractures, i.e. AO/OTA 43-A1 (20\%), A2 (50\%) and A3 (30\%), as compared to a study done by Wasim Akram et al. where they had A1 (25\%), A2 (55\%), A3 (20\%) which signifies the predominance of type A2 fractures in extraarticular distal tibial fractures. 19 patients $(63.33 \%)$ were males and $11(36.66 \%)$ were females, $18(60 \%)$ had right leg involved and 12(40\%) had left leg involved and a similar male sex predominance was seen in other studies.

21 patients $(70 \%)$ had RTA, 9 patients $(30 \%)$ had fall from height, out of nine patients with fall from height 5 were painters by occupation and a comparable result was found ina study done by Arjun ballal et al., where they had $85 \%$ RTA and $15 \%$ as fall from height as mode of injury which further shows us the role of RTA in Pilon A fractures. Average fracture healing time in our study was 18.7 weeks as compared to 21 weeks seen in a study done by Siddhartha Venkata et al. one of the reasons for this might be the predominance of young population with limited co morbidities that could effect the fracture healing. Average time taken for weight bearing is 18.6 weeks in our study as compared to 16 weeks in a study done by Harris et al., this difference might be attributed to education literacy level of patients as majority patients in our study came from rural background and could not understand meaning of tolerated and partial weight bearing, so to be on the safer side we did not allow weight bearing until we were confirmed of the fracture union. Coming to complications patient no.13 had wound infection which was initially superficial later became deep so implant had to be removed, after multiple debridement's flap cover was done which took up well, fracture united at 24 weeks and pt started tolerated weight bearing by 28 weeks. Compared to a study done by Wasim $e t$ $a l$., who had 5 infections out of 35 patients, this might be attributed to strict aseptic OT and ward measures taken out at our institution.

Patient no 14 and 18 had stiff ankle at the end of follow up with LEFS score of was 30 and 20 respectively, further they were advised physiotherapy for improving the movements, patient 14 was a a chronic alcoholic who did not follow our instructions of passive/active ankle mobilization post op which might have lead to restricted movements at the time of follow up, patient 16 was a old lady with co morbidities who could not follow our instructions properly.

Delayed union was seen in patient 1 who had metaphyseal communition and did not show signs of union at 8 weeks, he was further treated with autologous bone grafting and his fracture united at 25 weeks post op, he had a LEFS score of 30 and moderate range of motion, this is comparable to a study done by anil $\mathrm{k}$ jain et al. where they had 3 cases of delayed union out out of 21 who also had metaphyseal communition confirming that patients with severe metaphyseal communition do take time for healing.

\section{Clinical Photographs - Case 3}

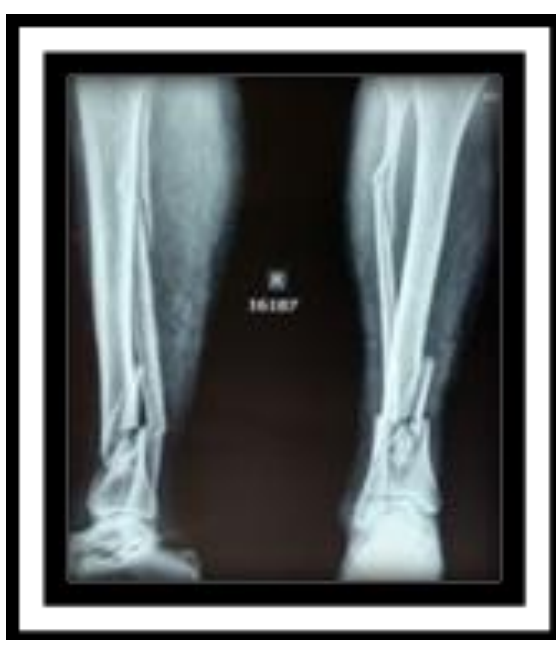

Fig 1: PRE OP X RAY

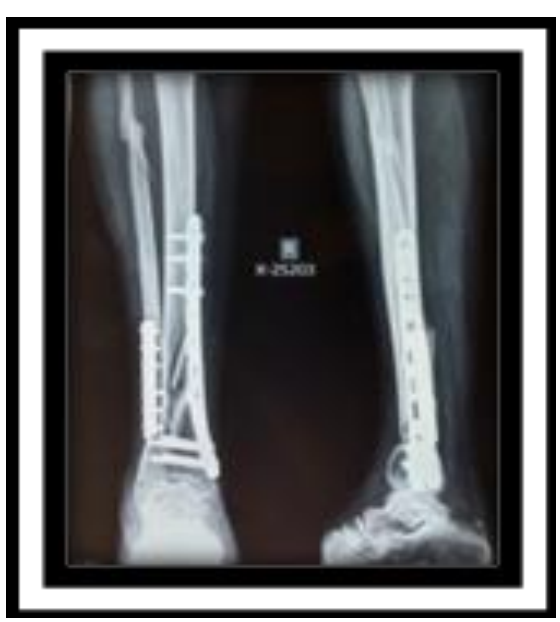

Fig 2: 6 Weeks POST OP 


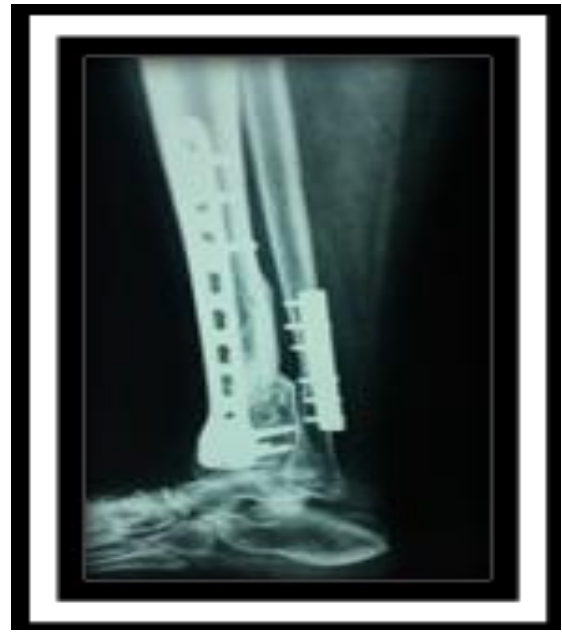

Fig 3: 20 Weeks POST OP

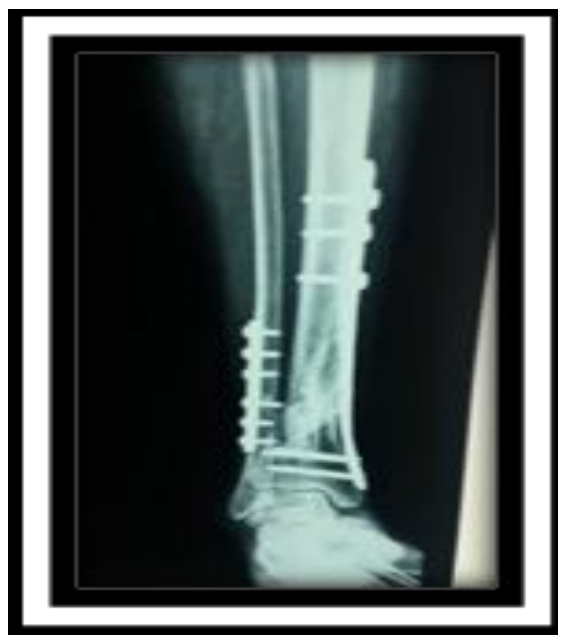

Fig 4: 20 WEEKS POST OP

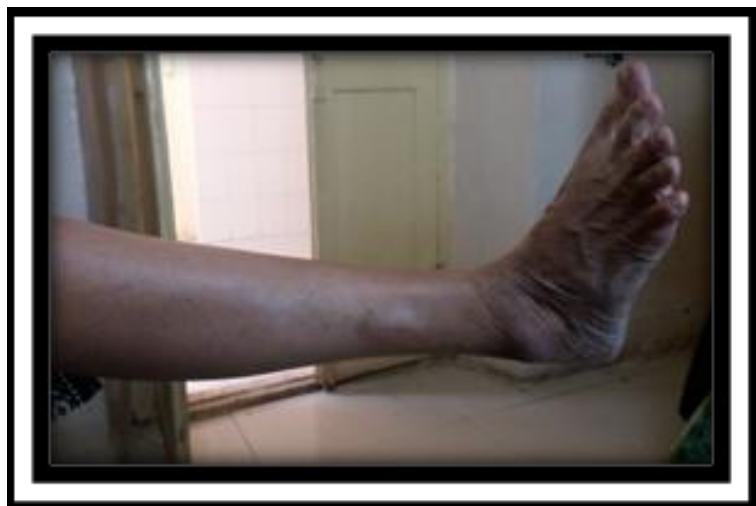

Fig 5: Dorsiflexion

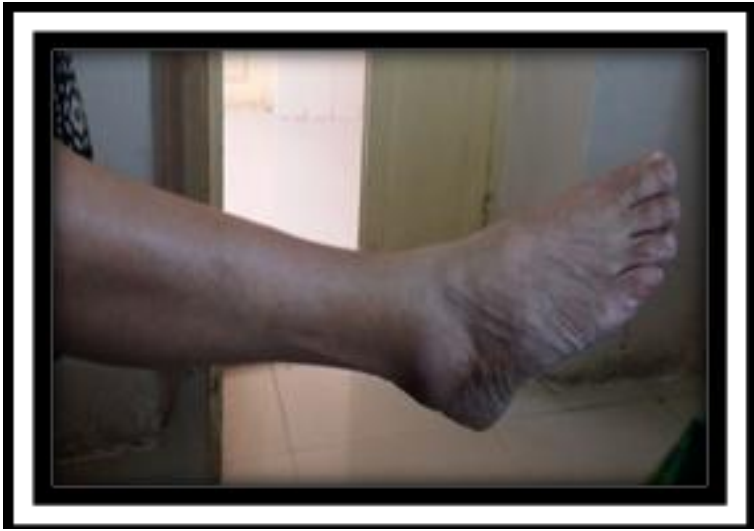

Fig 6: Plantarflexion
Case 17

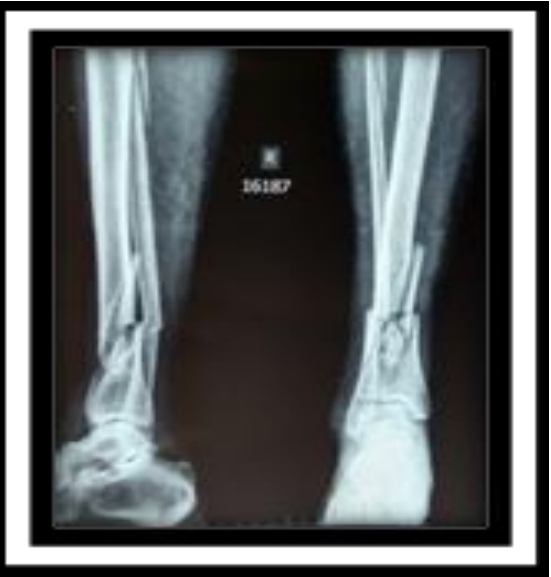

Fig 7: Pre Op Xay

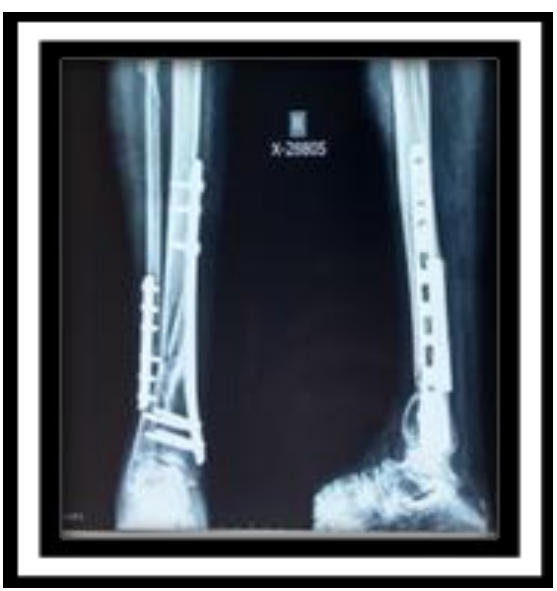

Fig 8: 5 Weeks Post Op

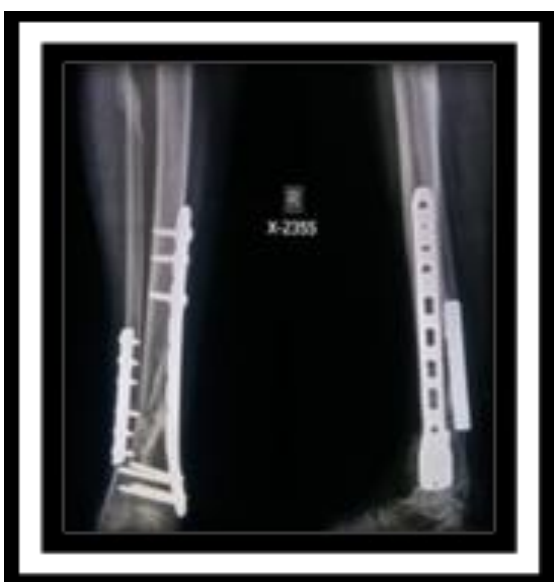

Fig 9: 23 Weeks

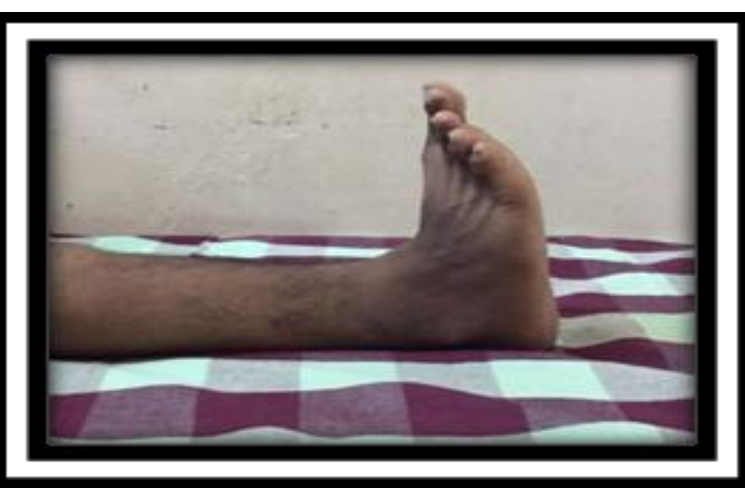

Fig 10: Dorsiflexion 


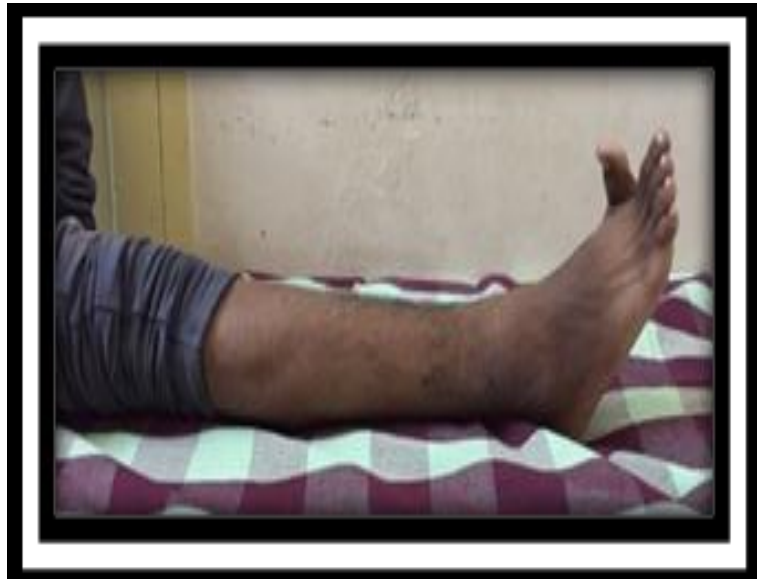

Fig 11: Plantarflexion

Case 1 (Delayed Union)

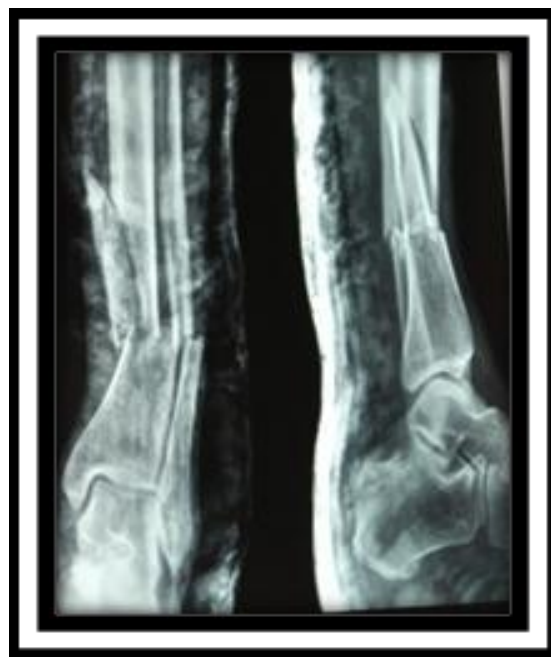

Fig 12: Pre OP X Ray

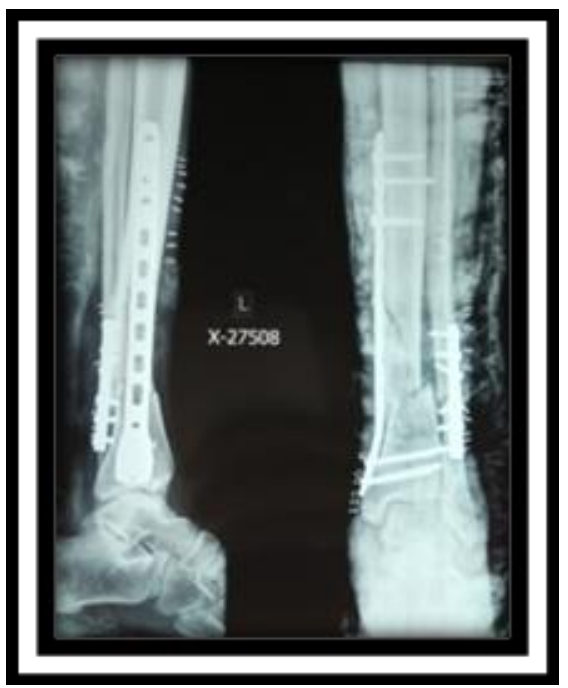

Fig 13: POST OP

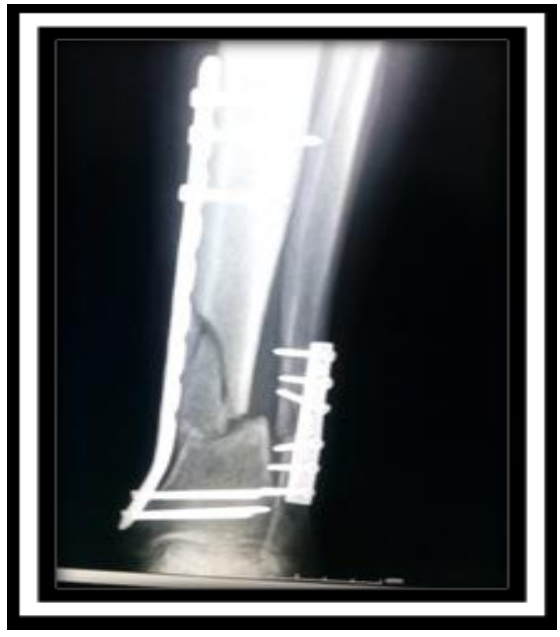

Fig 14: 2 Months Post Op With No Signs Of Union

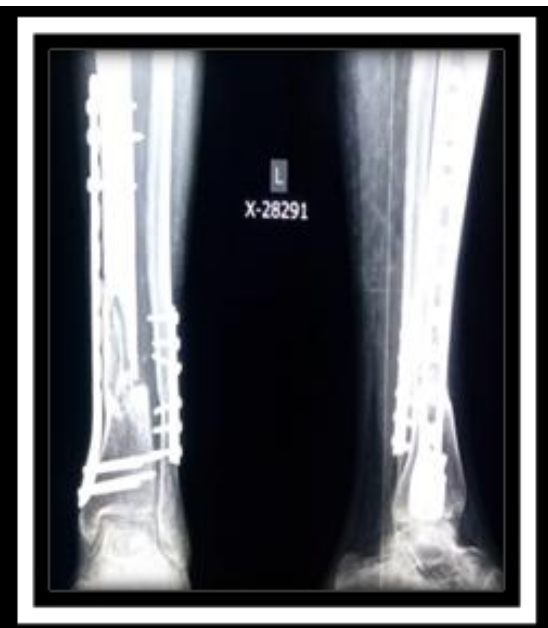

Fig 15: 5 Months Post Op after Bone Grafting Showng Signs of Union

Case 5

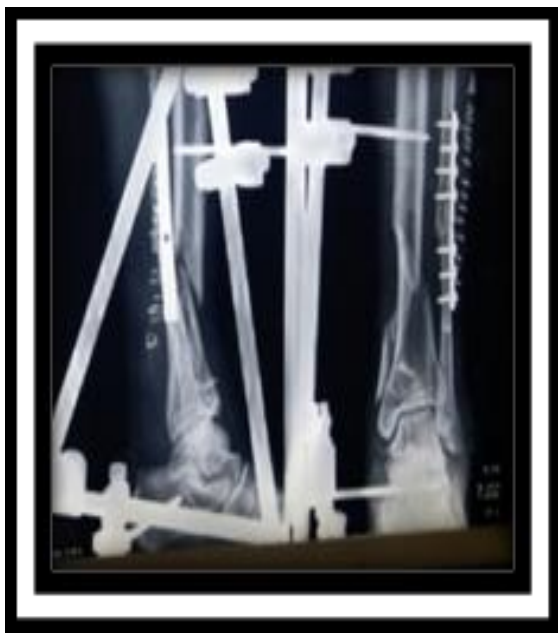

Fig 16: Patient Initially Placed On Ex Fix And Fibula Fixation Done Due To Poor Skin Condition. 


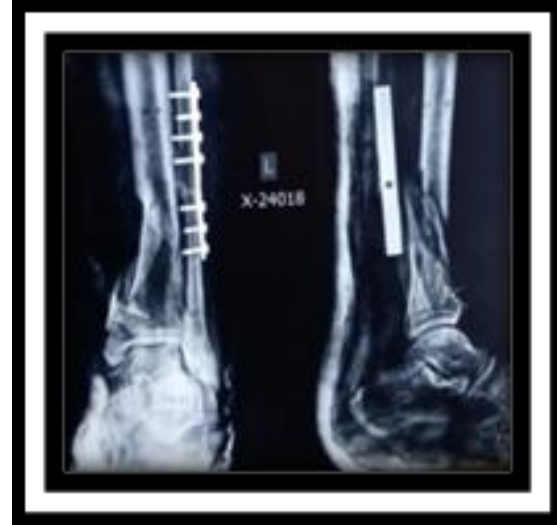

Fig 17: 2 Weeks After Removal Of Ex-Fix

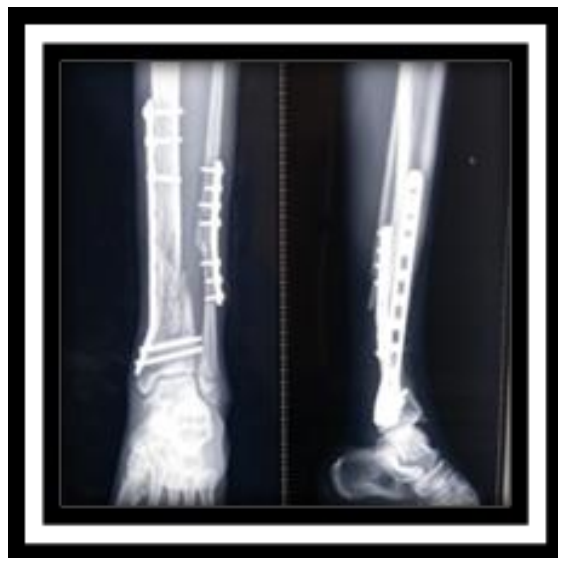

Fig 18: Mippo Dtlcp Was Done After Ruling Out Infection From Pin Tract Sites

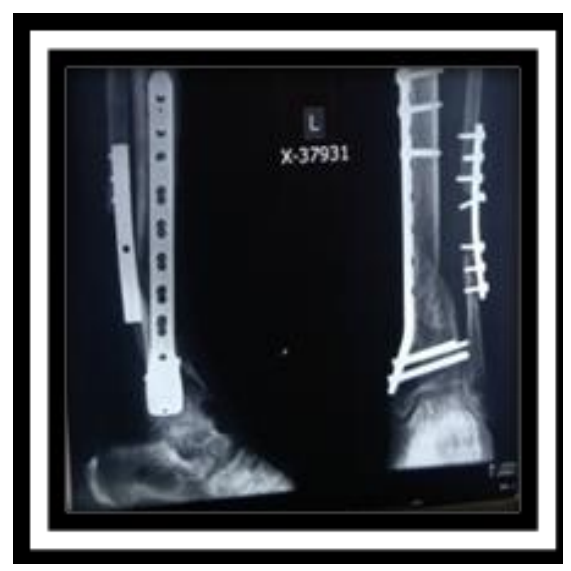

Fig 19: 20 Weeks Post OP

Case 13 (Infection)

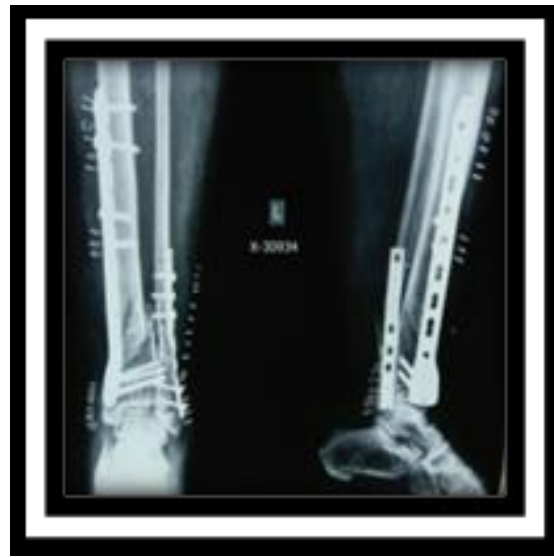

Fig 20: Immediate Post Op X Ray

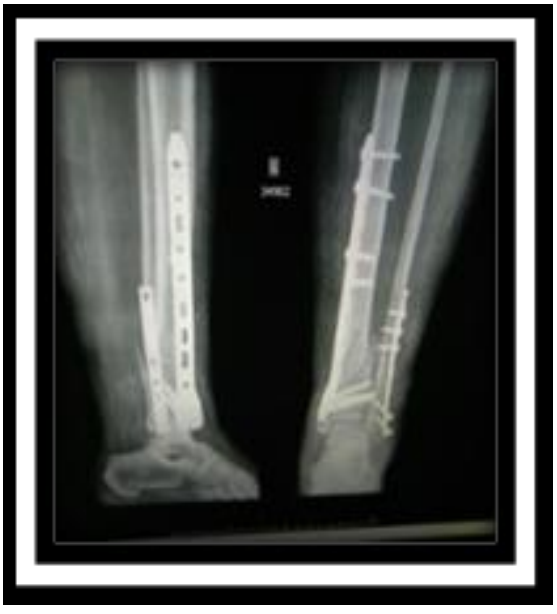

Fig 21: 6 Weeks Post OP

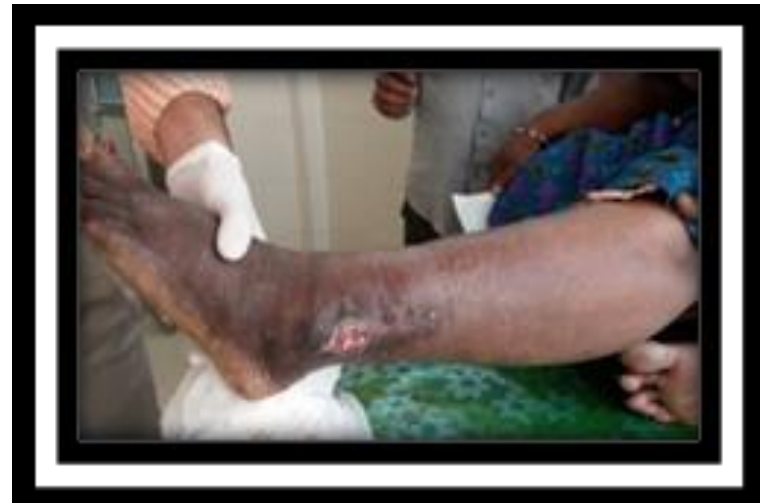

Fig 22: Patient Developed Wound Infection 3 Weeks Post Op

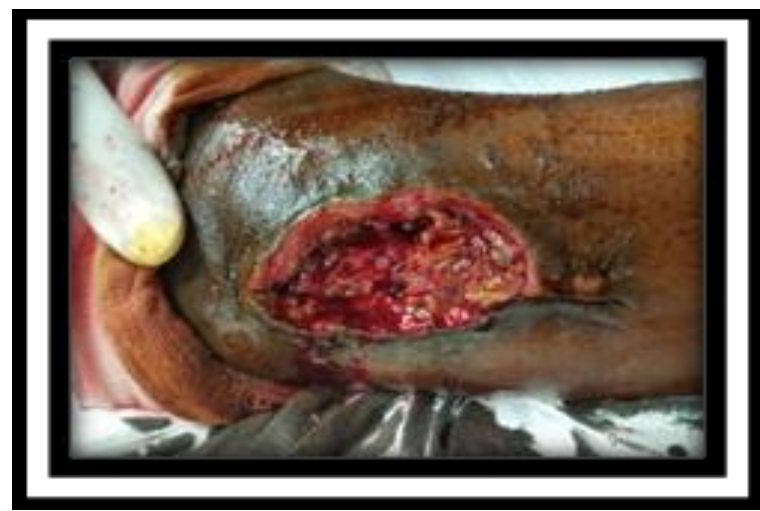

Fig 23: Post Wound Debridement

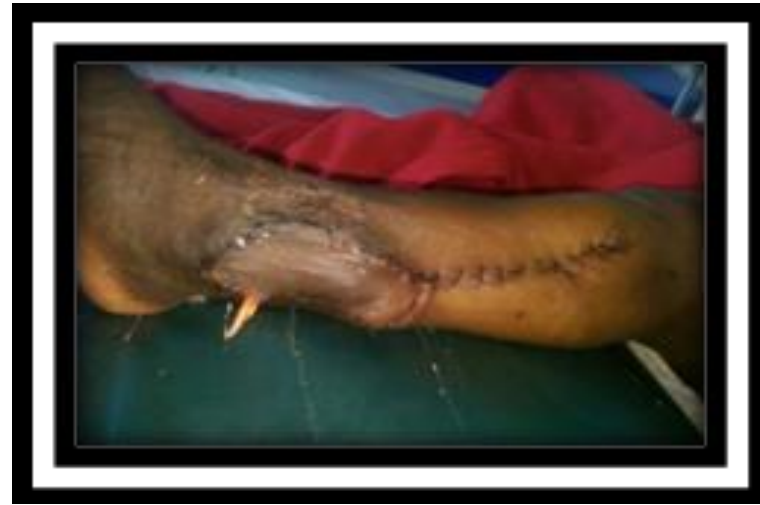

Fig 24: Post Flap Surgery

\section{Discussion}

Pilon fractures or distal tibia fractures are one of the complex injuries associated with significant soft tissue damage because 
of subcutaneous nature of bone and high velocity trauma associated with these injuries. $\mathrm{N}$ When soft tissue damage is significant, bridging external fixator is considered as superior option as it decreases the excessive soft tissue stripping and provides a temporary stable fixation to skeletal component. Definitive fixation can be planned after resolution of edema and wrinkling of skin is evident. In case of open fractures primary care is to provide wound debridement, tetanus immunization and broad spectrum antibiotic for prevention of infection is the main aim. During high velocity trauma the initial injury causes massive soft tissue damage which devitalizes the tissue around the fracture site ${ }^{[12]}$ Open reduction and internal fixation has shown increased rates of deep infection and wound dehiscence are the major soft tissue complications. The rate of infection is drastically decreased with the use of minimally invasive percutaneous plate osteosynthesis (MIPPO) in comparison with ORIF ${ }^{[13]}$. This is reflected in our results with only one case of superficial wound infection which resolved with antibiotics. Reports suggest that intramedullary nailing for A043A fractures has lowest infection rates compared to other procedures, but the technique is associated with other complications like Angular malunion, fat embolism syndrome, compartment syndrome and anterior knee pain. The studies of external fixator showed complications like imperfect reduction, loosening, malunion and pin tract infection. Open reduction with fixation of plates has reduced rates of angular malunion when compared to external fixation or intramedullary nailing but has disadvantage of high incidence of infection ${ }^{[14]}$. AO distal tibial locking plate using MIPPO technique provide a stable and rigid fixation of fractures minimizes the angulations and malunion without wound complication as in ORIF. The distal tibia plate is precontoured to the anatomy of distal tibia and thus allows placement of plates without disruption of fracture fragments. The distal end of plate allows placement of locking screws that provide stability. The proximal combination holes allow placement of locking or cortical screws where relative or absolute stability can be achieved. Acceptable degree of alignment is 5 degree of varus/valgus and 10 degree of recurvatum or procurvatum. All of our patients were found to have acceptable reduction and alignment post operatively and at union except one. Average fracture healing time was 18.8 weeks postoperatively. Associated fibula fracture was fixed with $\mathrm{i} / 3^{\text {rd }}$ tubular plate. As it is a rigid fixation, post operative plaster immobilization was not necessary and ankle stiffness was not a problem except in 2 patients. Weight bearing was delayed till radiological callus was visible on post operative check $\mathrm{x}$ ray and was started on average 12 weeks post op when the RUST score was 8 and partial weight bearing started around 6 weeks. Our results are comparable to other studies using MIPPO technique. In the present study, the mean age of 36 years is comparable to the studies by Bahari et al. and Redfern, Syed, Davies. The age of the patient had no bearing on the time to union in our study and may be the quality of bone and amount of soft tissue damage is an important factor in prognosis. In our study we had extra-articular fractures, i.e. AO/OTA 43-A1 (20\%), A2 (50\%) and A3 (30\%) which is comparable to Type AO A1(25\%), A2 (30\%), A3 (16.66\%) found in a study done by Hazarika et al. who also had predominance of A2 fracture pattern. Our study had one case of malunion out of 30 which is better than the above studies, this may be attributed to adequate reduction, plate countering and fixation with proper screw placement done in our study.

The mean time to union in our study was 18.7 weeks which is marginally better than 21.1 weeks this was obtained in a study done by Siddhartha Venkata Palisade M.S. (Ortho), Hitesh Lal M.S. (Ortho) et al. In the present study, 1 patient (3\%) had post-operative superficial wound infection and case healed after treatment with appropriate antibiotics and aseptic dressings and union in this patient was 22 weeks. The infection rate in the current series is better than a study done by Siddhartha Venkata Paluvadi M.S. (Ortho), Hitesh Lal M.S. (Ortho) et al. who had 4 infections in 50 patients $(10 \%)$,confirming about the aseptic and sterile techniques used at our centre.

Hazarika et al. treated 20 patients who had closed distal tibia fractures with minimally invasive locking plate osteosynthesis (MIPPO). Average time to full weight bearing was 18.1 weeks which was comparative to our study where we had average weight bearing time of 18.7 weeks, which further signifies distal tibia needs on an average 16 to 20 weeks for fracture union. Majority of patients we were able to mobilize post op $3^{\text {rd }} / 4$ th day non weight bearing with help of crutches and patient allowed tolerated and later partial weight bearing as radiological and clinical union progressed and full weight bearing when union was confirmed. Comparing the results with other methods of fixation our results are better when compared to ORIF and external fixation. All patients returned to work in 6 months and 80 percent had pre injury status by 6 months.

\section{Conclusion}

1. Locking compression plate is a good fixation system for Extraarticular distal tibia (Pilon A) fractures.

2. As surgical dissection is kept to minimum operating time is reduced.

3. The device provides good angular stability by its triangular reconstruction principle and thus helps in early mobilization.

4. One of the greatest advantage is in osteoporotic fractures where it may provide a solution to age old problem of screw cutout, late collapse and malalignment since the stability of construct doesn't entirely depend on the quality of the bone.

5. LCP by MIPPO technique leads to less periosteal stripping and less soft tissue damage and hence more rate of union and less chance of infection

\section{Reference}

1. Topliss CJ, Jackson M, Atkins RM. Anatomy of Pilon Fractures of Distal Tibia. J Bone Joint Surg Br 2005; 87:692-697

2. Moore KL. Clinically Oriented Anatomy. $2^{\text {nd }}$ Editon. Baltimore: Williams And Wilkins, 1985

3. Mehmet at Erol. The Results of Minimally Invasive Percutaneous Plate Osteosynthesis in Distal and Diaphyseal Tibial Fractures. Acta Ortho Traumatol Turc. 2012; 46(3):161-167.Doi:10.3944/AOTT.2012

4. Guo JJ, Tang N, Yanh HL, Tang TS. A Randomised Pilot Trial of Locking Plates Fixation Versus Intramedullary Nailing For Extraarticular Fractures of Distal Tibia.Bone And Joint Surg May. 2012; 94-B:704-708

5. Ghulamshabbir, Shujaat Hussain, Zulfiqar Ahmed, Kamran Shafi Minimally Invasive Osteosynthesis of Closed Fractures of Distal Tibia, J Ayub Medical College, Abottabad. 2011, 23(2).

6. Syah Bahari, Brain Lenehan, Hamad Khan, John P, Mcelwain Minimally Invasive Percutaneous Plate Fixation of Distal Tibia Fractures Acta Orthop. Belg. 2007; 73:635-640 
7. Jen Francois, Geoffrey Vandeputte, Frank Verheyden, Guy Nelen Percutaneous Plate Fixation Of Fractures Of Distal Tibia ACTA Orthop, Belg. 2004; 70:148-154

8. Oliver Borens. Peter Rloen, Jeffrey Richmond, Goetz Roederer, David Levine, Minimally Invasive Treatment For Tibial Plafound Fractures With Low Profile Plating. Priliminary Result of 17 Cases. Pg No 24-26

9. Mario Ronga, Umile Giuseppe Longo and Nicola Maffuli. Minimally Invasive Locked Plating Distal Tibia Fractures Is Safe And Effective. Clinical Orthopaedics and Related Research. 2010; 468(4):975-982

10. Abid Mushtaq, Rizwan Shahid, Muhammed Asif, Mohammed Maqsood. Distal Tibia Fracture Fixation with Lcp Using Mippo; European Journal of Trauma and Emergency Surgery 2009; 35(2).

11. Blauth M Bastinl, Krettek C, Knopc, Evans. Surgical Options For The Treatment Of Severe Tibial Pilon Fractures. A Study of Three Techniques. J Orthop Trauma. 2001; 15:153-160.

12. Bonar SK, Marsh JL. Tibial Plafound Fractures; Changing Principles of Treatment. J Am Acad Orthop Surg 1994; 2:297-305

13. Borelli J, Prikket W, Becker D, Ricci W. Extraosseus Blood Supply of The Distal Tibia And Effects Of Different Plating Techniques. Human Cadaveric Study. J Orthop TRAUMA 2002; 16:691-695.

14. Ch. Banikanta Sharma, Sanjib Waukhom. Management of Fractures of Distal Tibia with Locking Medial Metaphyseal Plate. Journal Of Evidence Based Medicine And Healthcare; 2015; 2:4209-4212

15. Anil K Mishra, Chalise PK, Shah SB, Adhikari V, Singh RP. Outcomes of Minimally Invasive Percutaneous Plate Osteosynthesis in Closed Fractures of Distal Tibia. Journal of College of Medical Sciences. 2013; 9(2):3844. 